\title{
Anatomical variations in atrioventricular conduction system with reference to ventricular septal defects
}

\author{
R. A. Latham ${ }^{1}$ and R. H. Anderson \\ From the Institute of Child Health, University of Liverpool; \\ and Department of Anatomy, University of Manchester
}

The course of the atrioventricular conducting tissues was studied in 1o hearts having ventricular septal defects. Seven of the defects were in the membranous portion of the septum, and in these hearts the common atrioventricular bundle passed down the posterior edge of the defect and bifurcated on its inferior border. In 3 of these specimens the bundle deviated to the left side of the septum before bifurcating. The 3 remaining hearts possessed defects of the muscular septum. In 2 of these the common bundle bifurcated within the septum anterosuperiorly to the defect. The left branch descended to become intimately related to the anterior border of the defect. In the other heart with low anterior intramuscular defects the right bundle-branch traversed the septum and divided astride the defect. These results are discussed in relation to surgical repair of the defects and to the reports of previous investigators. The added risk of damaging the conducting tissue is emphasized in cases which deviate from the usually accepted pattern.

Previous studies on hearts with intramembranous defects of the ventricular septum have emphasized the relation between the atrioventricular bundle and the posteroinferior border of the defect (Reemtsma and Copenhaver, I958; Reemtsma, Copenhaver, and Creech, 1958; Truex and Bishof, 1958; Lev, 1960; Titus, Daugherty, and Edwards, 1963a). While Lev (1960) suggested that the bundle may be related to intramuscular defects, the general inference was that these were unlikely to be intimately involved with the conducting tissue. Our attention was drawn to this problem in the course of a histological study of a heart in which an intramuscular defect had been repaired. We were surprised to find that the right bundlebranch had been extensively traumatized, and that the left branch passed down the anterior border of the defect. It was therefore decided to reinvestigate the course of the atrioventricular specialized tissue in relation to varying types of interventricular septal defects.

\section{Materials and methods}

The hearts used in this investigation were taken from the cardiopathological collection of the

1 Present address: Dental Research Center, University of North Carolina, Chapel Hill, U.S.A.
Royal Liverpool Children's Hospital. Ten hearts containing varying interventricular septal defects were studied. In addition, three hearts without defects were examined for comparative purposes. The type of defect present in the hearts is indicated in the Table.

The hearts were first photographed, and then a block of tissue was dissected out containing the atrioventricular septum from the coronary sinus to the defect. This block was then rephotographed from right and left aspects, before dehydration and embedding in paraffin wax. Serial sections were cut at $10 \mu$ and several sections in every 50

T ABLE Defects in hearts studied

\begin{tabular}{|c|c|c|c|c|}
\hline \multirow{2}{*}{$\begin{array}{l}\text { Specimen } \\
\text { No. }\end{array}$} & \multirow{2}{*}{$\begin{array}{l}\text { Block } \\
\text { No. }\end{array}$} & \multirow[t]{2}{*}{ Age } & \multicolumn{2}{|c|}{ Type of defect } \\
\hline & & & $\begin{array}{l}\text { Intra- } \\
\text { mem- } \\
\text { branous }\end{array}$ & $\begin{array}{l}\text { Intra- } \\
\text { muscular }\end{array}$ \\
\hline 782 & 108 & $5 \mathrm{mth}$ & & $\mathbf{x}$ \\
\hline $73 I$ & IIO & $22 \mathrm{dy}$ & $\mathbf{x}$ & \\
\hline 686 & I I I & 27 dy & $\mathbf{x}$ & \\
\hline 612 & II2 & I yr $5 \mathrm{mth}$ & $\mathbf{x}$ & \\
\hline 794 & 113 & $12 \mathrm{wk}$ & $\mathbf{x}$ & \\
\hline 798 & 114 & $5 \frac{1}{2} \mathrm{yr}$ & $\mathbf{x}$ & \\
\hline 197 & 116 & $4 \mathrm{mth}$ & $\mathbf{x}$ & $\mathbf{x}$ \\
\hline 226 & 117 & Io wk & $\mathbf{X}$ & \\
\hline 647 & 118 & $2 \mathrm{yr} 8 \mathrm{mth}$ & $\mathbf{X}$ & \\
\hline 587 & II9 & $2 \mathrm{yr}$ I mth & & $\mathbf{x}$ \\
\hline
\end{tabular}


were mounted and stained with Masson's trichrome technique. The intervening sections were stored and, after examination of the mounted sections, further sections were mounted and stained as required. In some cases adjacent sections were stained by the PAS technique, after blockade in 5 per cent dimedone, to show glycogen (Bulmer, r959).

\section{Results}

It was relatively easy to define histologically the atrioventricular bundle and its branches. The tracts of specialized cells were surrounded by connective tissue and were well demarcated from the adjacent myocardium. The sections processed to show glycogen revealed little difference between the specialized cells and myocardial cells. In size, the specialized cells were marginally larger than ventricular myocardial cells, and exhibited cross-striations. No cells were identified in the sections examined which corresponded to the 'Purkinje' cells seen in artiodactyla except in poorly fixed tissue.

The course of the specialized tissue was found to vary according to the type of defect. It was difficult to distinguish these defects on any strict macroscopical criteria, but 7 were designated as within the membranous portion of the septum while 3 were intramuscular. The intramembranous defects were generally more anterior than the muscular type, closer to the atrioventricular ring, and higher in relation to the crista supraventricularis.

a) Intramembranous defects Two groups were identified according to the course of the atrioventricular bundle.

Five hearts were virtually identical and constituted the first subgroup. These were No. 226, 612, 647, 686, and 731 in the Royal Liverpool Children's Hospital collection (see the Table). The atrioventricular bundle was found to pierce the fibrous annulus in a normal fashion. As it reached the ventricular septum the bundle came into contact with the defect and descended along its posterior edge. Close to the inferior edge of the defect the bundle began to bifurcate. The left bundle-branch was an extensive structure which passed from the bifurcation along the length of the inferior edge of the defect and descended subendocardially on the left side of the septum. The right branch was a thick cord given off the bifurcation near the middle of the inferior edge of the defect. This structure passed intramyocardially to emerge in the subendocardium near the anterior papillary muscle of the tricuspid valve. The arrangement of specimen 686 is shown in Fig. I, 2,
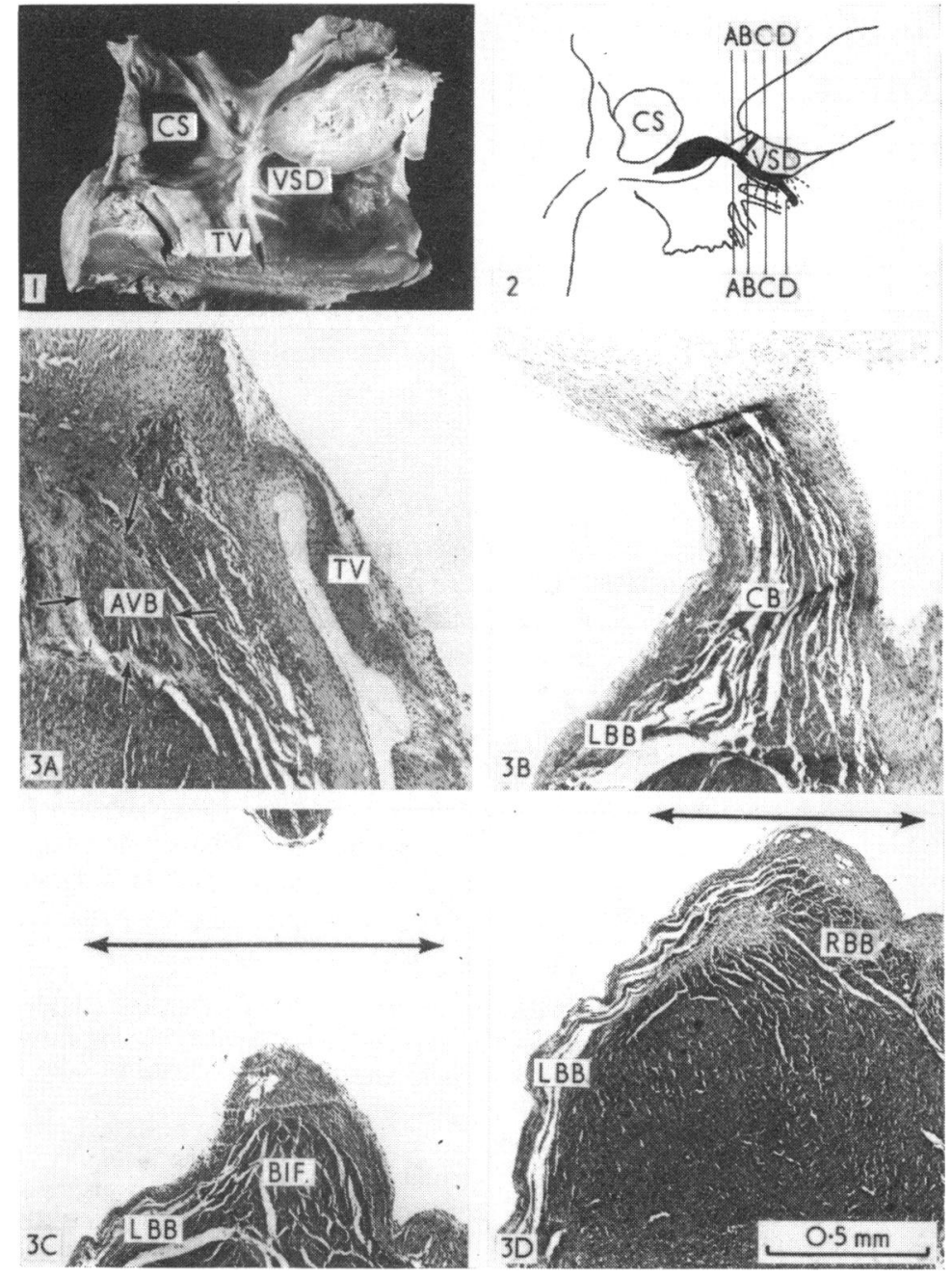

FI G. I Block II I from specimen 686, showing intramembranous septal defect (VSD), opening of left superior vena cava (CS), and tricuspid valve $(T V)$.

FIG. 2 Reconstruction of conducting tissue on tracing of Fig. $I$.

FIG. 3 A-D Four representative sections at levels $A-D$ are shown. $A V B$, atrioventricular bundle; $C B$, common bundle; BIF, bifurcation; $\angle B B$ and $R B B$, left and right bundle-branches; TV, tricuspid valve. The arrow is in the defect. All are at the same magnification as shown by $0.55 \mathrm{~mm}$ line in $3 D$.

and 3 A-D. Specimen No. 647 varied slightly in that an interatrial septal defect was also present. In this case the bundle deviated to the left after piercing the annulus and continued 
along the left side of the bar of tissue between the defects until it reached the ventricular defect. It then bifurcated on the posteroinferior edge in the manner described above.

The second subgroup comprised specimens 794 and 798. The bundle descended onto the posterior edge of the defect as described, but then deviated to the left. It then passed an appreciable distance down the left side of the septum before bifurcating on the inferior border of the defect. The left branch spread out subendocardially while the right branch, again cord-like, ascended through the myocardium of the septum to emerge near the anterior papillary muscle (Fig. 4-7).

b) Intramuscular defects Three hearts were examined with defects of this type. In No. 197 and 782 the defect was close to the atrioventricular ring, simulating the intramembranous type. In the third heart (No. 587) there were two defects, both remote from the AV ring. The conducting tissue followed different routes in each case.

Specimen No. 197 was further complicated, since histological examination revealed the presence of a small intramembranous defect. The bundle passed posteroinferiorly to this, as in the other fibrous defects, but did not bifurcate. Bifurcation occurred in the same fashion as that illustrated for No. 782 in Fig. 8, 9, and ro A-D. This specimen was the first heart examined in this series and it revealed widespread trauma to the right bundlebranch in front of the defect. The atrioventricular bundle pierced the annulus in the normal fashion before the bundle entered the muscular septum. In this position the right half was infiltrated with haemorrhagic exudate, though there was no evidence of direct trauma. The bundle passed through the septum to a point above the anterosuperior edge of the defect and bifurcated within the septum. The left branch immediately fanned out to form a typical subendocardial ribbon. The posterior fascicles of this branch passed directly onto the anterior edge of the defect, occupying its full thickness. On reaching the inferior edge they curved around it and then descended on the left side of the septum. The cord-like right branch was traced into a large haematoma present in the septum anterior to the defect and also involving the anterior papillary muscle. The terminations of the bundle in heart No. 197 showed identical relations to the muscular defect.

The remaining heart in this series, No. 587, had distal duplicated defects. The bundle in
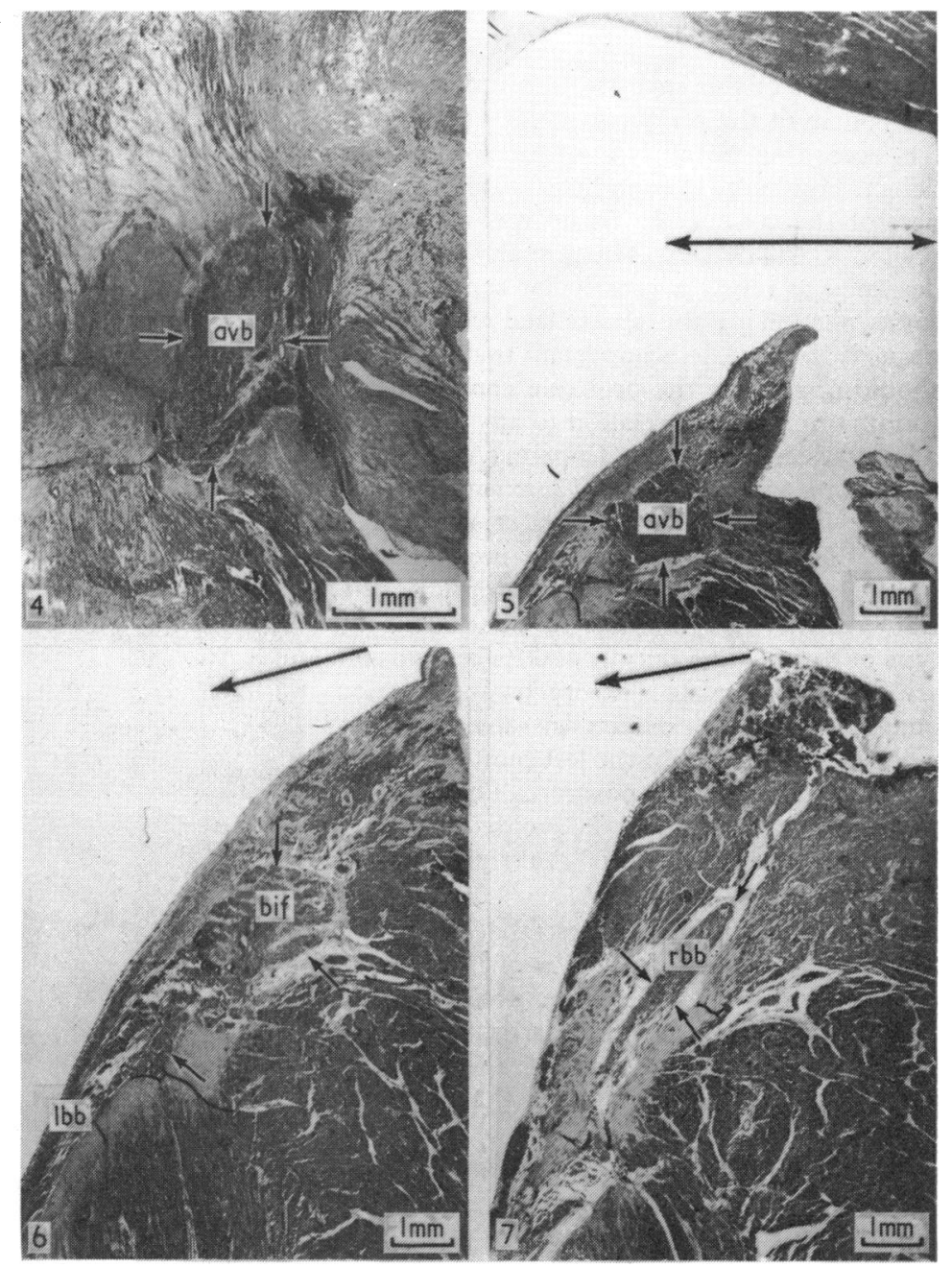

FIG. 4-7 Representative sections of specimen 798 with an intramembranous defect. The arrow indicates the defect. Abbreviations as before.

this case bifurcated normally on the crest of the septum and the left branch occupied the expected position. The cord-like right branch followed an extensive intramyocardial route beneath the anterior papillary muscle and emerged on the posteroinferior edge of the superior defect (Fig. I I and 12). It then bifurcated again in this position and the divisions passed down on both right and left sides of the septum.

Fig. I3 illustrates the varying routes taken by the conducting tissue with reference to the types of ventricular septal defect described. 


\section{Discussion}

The results of this investigation indicate that the course of the atrioventricular bundle differs according to the type of interventricular defect. However, in each heart studied, some part of the conducting tissue was intimately related to the defects. Thus, in any operation designed to repair a ventricular septal defect some portion of the specialized conducting tissue is 'at risk'. It is important that surgeons should appreciate the probable course of the conducting tissue in relation to any particular defect. Reemtsma and Copenhaver (1958), Reemtsma et al. (1958), Truex and Bishof (1958), Lev (1960), and Titus et al. (1963a) all studied high defects of the membranous portion of the interventricular septum. We can confirm their findings in this particular type of defect. The bundle and its bifurcation are intimately related to the posteroinferior quadrant of such a defect. In repairing such a defect from the right the left bundle-branch is in a relatively safe position. Thus, if the posteroinferior quadrant is avoided the only other structure at risk is the right bundle branch.

Our investigations suggest that injury of this intramyocardial fascicle is of importance not only with regard to the production of right bundle-block, as haemorrhagic exudate can apparently track in a retrograde direction along the conducting tissue. In the specimen described, injury of the right branch well down the septum produced haemorrhagic exudate in the parent bundle as it pierced the fibrous annulus. Thus, injury of the distal right branch could possibly produce complete atrioventricular dissociation by retrograde spread, producing oedema or fibrosis. Titus et al. (1963b) and McGoon, Ongley, and Kirklin (1964) pointed out that in patients suffering from AV dissociation it was rare to find evidence of direct trauma. This would also suggest that the bundle is likely to be sensitive to oedema or pericellular exudate. It therefore seems to be important to avoid the right bundle-branch if at all possible. In all the specimens presently studied with intramembranous defects this structure ran intramyocardially from the inferior edge of the defect towards the anterior papillary muscle. If this area is avoided during suturing, in addition to the posteroinferior quadrant, then damage to the conducting tissue should be avoided.

The variations noted from this pattern described in membranous defects were on the left side of the septum. These should make the bundle less at risk when repaired from the
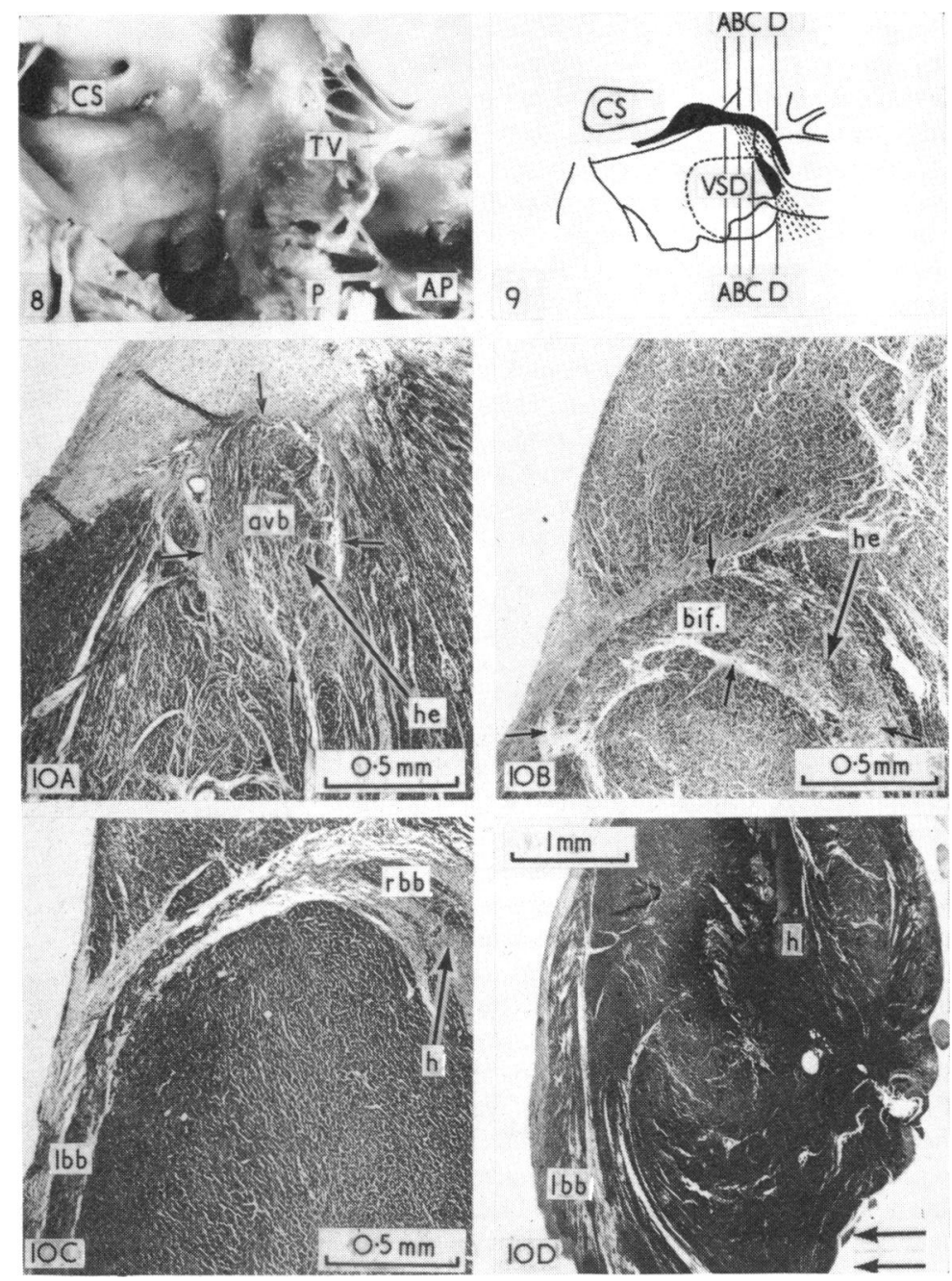

FIG. 8 Block 108 from Specimen 782.

Abbreviations as before; $P$, patch on defect; $A P$, anterior papillary muscle.

FIG. 9 Reconstruction of conducting tissue on tracing of Fig. 8.

FIG. IO A-D Four representative sections at levels $A-D$. Abbreviations as before; he, haemorrhagic exudate; $h$, haematoma. The two arrows indicate the anterior extent of the defect.

right. The right bundle-branch followed the same intramyocardial course in these hearts also, so avoidance of the area above the anterior papillary muscle should spare the conducting tissue in these patients.

We found a relation between intramuscular defects and the conducting tissue. This is 
quite different from the arrangement in membranous defects, and it is important that the difference should be noted. This is particularly so in defects near the atrioventricular ring which resemble membranous defects on macroscopical examination. Previous workers found no relation between such defects and the conducting tissue. Lev (1960), however, inferred that the conducting tissue might be related to the anterior edge of a muscular defect. The present work presents evidence substantiating this inference. In both hearts studied where the muscular defect was near the ring, the bundle bifurcated above the anterosuperior quadrant of the defect. The left branch was intimately related to the anterior edge of the defect. The right branch passed intramyocardially towards the anterior papillary muscle, again anterior to the defect. Thus, in these hearts it is essential to avoid the anterosuperior and anterior edges of the defect, in contradistinction to avoiding the posteroinferior quadrant in muscular defects. It is therefore of some importance to distinguish intramembranous and intramuscular defects before starting a repair operation. In the present series the intramembranous defects were in all cases closer to the AV ring, higher in relation to the crista supraventricularis, and more anterior in the septum.

The other specimen studied with an intramyocardial defect was also instructive. This defect was distant from the AV ring and the bifurcation, yet the right branch ran a lengthy intramyocardial route before bifurcating on the inferior edge of the defect. Possibly this relation was coincidental, in that the defect was close to the anterior papillary muscle. However, in the absence of contrary studies it may be prudent to avoid the quadrant nearest the anterior papillary muscle in repair operations on such distal defects.

The described relation of the specialized conducting tissue to the differing types of defect is to be expected from its embryo-

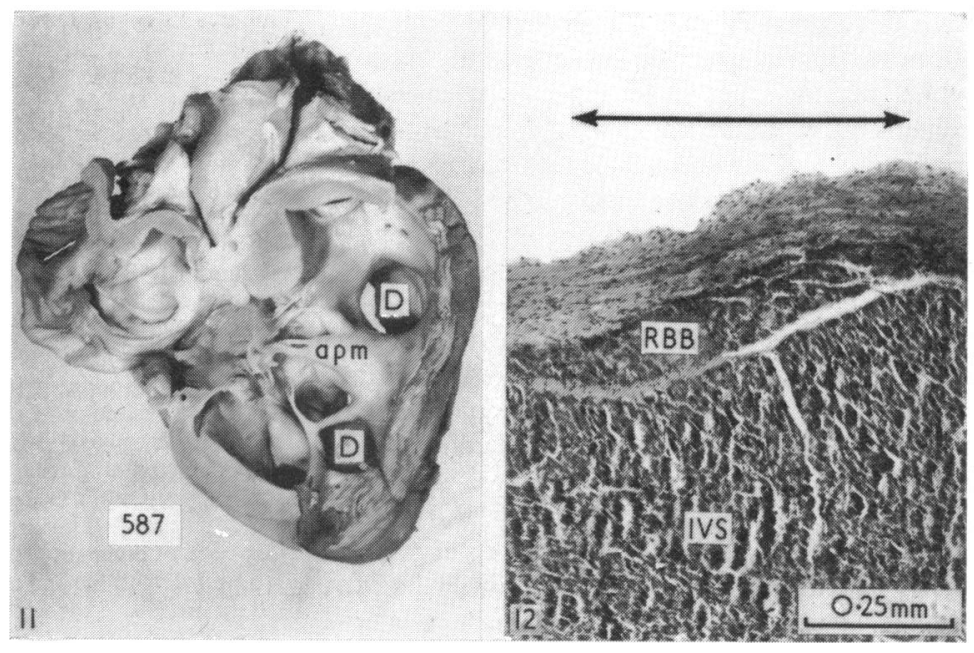

FIG. II Photograph of Specimen 587. D, defects; apm, anterior papillary muscle.

FIG. I2 Bifurcation of right bundle branch $(R B B)$ on the lower edge of superior defect (indicated by arrow). IVS, interventricular septum.

logical development. The bundle bifurcates astride the developing muscular portion of the interventricular septum, and below the portion formed from endocardial and bulbar musculature (Boyd, 1965). Thus, the specialized tissue would be situated below membranous defects but above muscular defects.

This arrangement would be found irrespective of the origin of the bundle. Walls (1947) considered that the bundle was formed by active migration. On this principle, cases such as that described by Huntingford (1960) are interpreted as representing postjunctional severing of the bundle. However, James (1970) opined that these cases were better explained by lack of union of the node and the bundle,

FIG. I3 Diagrams showing course of conducting tissue in relation to defects studied. $A, B-$ simple intramembranous defects. C-intramembranous defect associated with ASD. D, Eintramuscular defects.
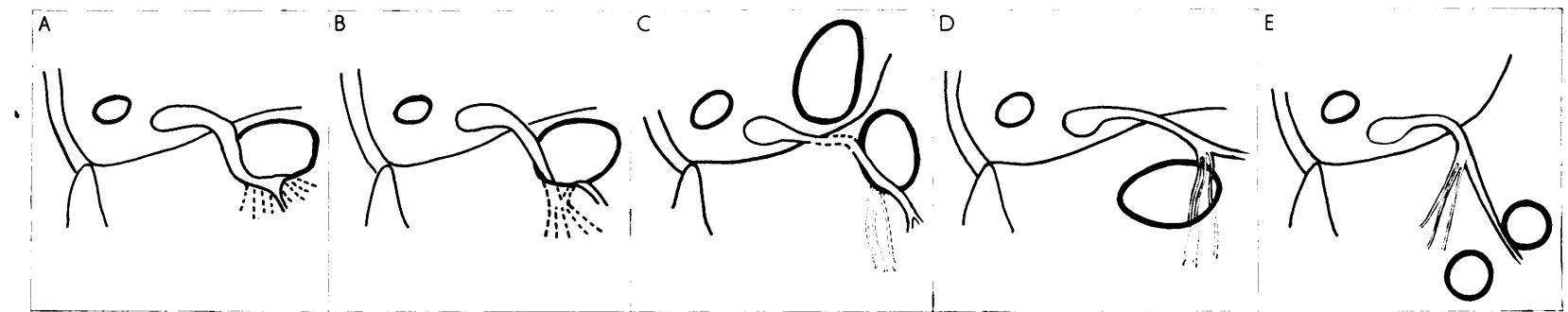
each developed in situ. Physiological findings support this contention (Lieberman, 1970) while recent studies of embryological and congenitally malformed specimens provide morphological evidence of its correctness (Anderson and Taylor, unpublished data).

A further point arising from this investigation is of morphological interest. None of the hearts studied showed cells that corresponded to the cells identified in artiodactyla by Purkinje (1845). Indeed, the specialized cells differed only slightly from ventricular myocardium, being recognized in collections rather than as single cells. Workers such as James (196r) used the presence of 'Purkinje' cells as criteria for recognizing conducting tissue in extranodal sites. On the other hand, Davies (1942) found no 'Purkinje' cells in the upper parts of the interventricular septum, a view endorsed by Copenhaver and Truex (1952) who were unable to identify such cells anywhere within the AV node. Truex (I96I) expanded this hypothesis and suggested that the presence of 'Purkinje' characteristics might be a consequence of fixation artefacts, since he noted segments of normal atrial cells possessing similar characteristics. He thought it probable that true 'Purkinje' cells existed only in artiodactyla. The present investigation supports his and previous workers' views with regard to the human heart. This point and others with regard to 'Purkinje' cells were discussed more fully in a paper relating to the architecture of the AV node in the hearts presently studied (Anderson and Latham, I97I).

The authors wish to thank Dr. R. S. Jones for his interest in this work, Mrs. A. Smith for histological work, and Messrs. K. Walters and R. Wylie for photomicrography.

\section{References}

Anderson, R. H., and Latham, R. A. (1971). The cellular architecture of the human atrioventricular node, with a note on its morphology in the presence of a left superior vena cava. Fournal of Anatomy. In the press.

Boyd, J. D. (1965). Development of the heart. In Handbook of Physiology. Section 2: Circulation, Vol. 3, pp. 25II-2543. Ed. by W. F. Hamilton. American Physiological Society, Washington.
Bulmer, D. (1959). Dimedone as an aldehyde blocking reagent to facilitate the histochemical demonstration of glycogen. Stain Technology, 34, 95.

Copenhaver, W. M., and Truex, R. C. (1952). Histology of the atrial portion of the cardiac conduction system in man and other mammals. Anatomical Record, 114, 601.

Davies, F. (I942). The conducting system of the vertebrate heart. British Heart fournal, 4, 66.

Huntingford, P. J. (1960). The aetiology and significance of congenital heart block. Fournal of Obstetrics and Gynaecology of the British Empire, 67, 259.

James, T. N. (196I). Morphology of the human atrioventricular node, with remarks pertinent to its electrophysiology. American Heart fournal, 62, 756.

James, T. N. (1970). Cardiac conduction system: fetal and postnatal development. American fournal of Cardiology, 25, 213.

Lev, M. (1960). The architecture of the conduction system in congenital heart disease. III. Ventricular septal defect. Archives of Pathology, 70, 529.

Lieberman, M. (1970). Physiologic development of impulse conduction in embryonic cardiac tissue. American fournal of Cardiology, 25, 279.

McGoon, D. C., Ongley, P. A., and Kirklin, J. W. (1964). Surgical heart block. American fournal of Medicine, 37, 749.

Purkinje, J. E. (1845). Mikroskopisch-neurologische Beobachtungen. Archiv für Anatomie, Physiologie und wissenschaftliche Medicin, 12, $28 \mathrm{I}$.

Reemtsma, K., and Copenhaver, W. M. (1958). Anatomic studies of the cardiac conduction system in congenital malformation of the heart. Circulation, 17, 271.

Reemtsma, K., Copenhaver, W. M., and Creech, O. (1958). The cardiac conduction system in congenital anomalies of the heart: studies on its embryology, anatomy, and function. Surgery, 44, 99.

Titus, J. L., Daugherty, G. W., and Edwards, J. E. (I963a). Anatomy of the atrioventricular conduction system in ventricular septal defect. Circulation, 28, 72.

Titus, J. L., Daugherty, G. W., Kirklin, J. W., and Edwards, J. E. (1963b). Lesions of the atrioventricular conduction system after repair of ventricular septal defect; relation to heart block. Circulation, 28, 82.

Truex, R. C. (196r). Comparative anatomy and functional considerations of the cardiac conduction system. In The Specialized Tissues of the Heart (Proc. of a Symposium, 1960, Rio de Janeiro), p. 22. Ed. by A. Paes de Carvalho, W. C. de Mello, and B. F. Hoffman. Elsevier, Amsterdam.

Truex, R. C., and Bishof, J. K. (1958). Conduction system in human hearts with interventricular septal defects. Fournal of Thoracic Surgery, 35, 421.

Walls, E. W. (1947). The development of the specialized conducting tissue of the human heart. Fournal of Anatomy, 81, 93.

Requests for reprints to Dr. R. H. Anderson, Anatomy Department, The University, Manchester, Mr3 9PL. 